examined for comparison in the normal state, with the field applied, and with same field in the superconductive state. The latter was prepared by (1) cooling in the steady field to the mixed state, and (2) cooling in zero field, and then switching on. The results for the alloy were encouraging. For procedure (1), the main Fourier component of the modulated count (corresponding to the frequency of the applied field) was observed to broaden upon cooling to $5.5 \mathrm{~K}$; upon further cooling to $3 \mathrm{~K}$, a bifurcation was resolved, and a satellite peak identified as arising from the saddle-point mentioned above. Procedure (2) however showed no such splitting, indicating that the structure was smeared out by the preparation. Less exciting results were found for the $\mathrm{Nb}$. No splitting was observed with either (1) or (2), but the main (external field) line appeared with much larger amplitude.

The authors conclude their report with a discussion concerning the possible diffusion of the $\mu^{+}$. Far from being simply trapped at a favourable location the tunnelling rate between $\mathrm{Nb}$ sites is estimated as being at least $10^{12} \mathrm{~s}^{-1}$. If the observed sharp line in $\mathrm{Nb}$ is attributed to motional narrowing (as occurs in NMR for example) a large diffiusion constant of the order $10^{-2} \mathrm{~cm}^{2}$ $\mathrm{s}^{-1}$ is needed. At present, actual diffusion mechanisms remain speculative and the authors plan further work to resolve them.

\section{Comet chemistry}

from $M . K$. Wallis

"The Study of Comets", The International Astronomical Union Colloquium No. 25, held from October 28 to November 1 at NASA's Goddard Space Flight Center in Maryland, was timed to take stock of new data from comet Kohoutek and also to precede critical decisions on a possible cometary space probe.

Most of the active comet researchers from the United States and Western Europe put in an appearance, but unfortunately, in spite of sponsorship from the IAU and the International Committee on Space Research, nobody from the strong Soviet and Czech groups attended.

Much interest was attracted by the reports of the new molecular emissions, revealed in the ultraviolet and radio spectra. The 1,304 and 1,657 $\AA$ multip. lets have given data on atomic oxygen and carbon, reported P. D. Feldman of Johns Hopkins University. And the radio observations, reviewed by $L$. $E$. Snyder (University of Virginia) have revealed $\mathrm{CH}, \mathrm{OH}, \mathrm{HCN}$ and $\mathrm{CH}_{3} \mathrm{CN}$ in comet Kohoutek and $\mathrm{H}_{2} \mathrm{O}$ in comet
Bradfield. Quantitative interpretation is thwarted however, as all except the HCN emission is pumped. The repeated failure to detect the predicted formaldehyde $\left(\mathrm{H}_{2} \mathrm{CO}\right)$ emission is also put down to pumping.

Surprisingly, there is also a new identification in the optical-of $\mathrm{H}_{2} \mathrm{O}^{+}-\mathrm{a}$ joint effort with P. Benvenuti (Asiago Observatory, Italy) and $P$. Wehninger (University of Tel-Aviv) as G. Herbig of Lick Observatory related. There are numerous lines from this ion, from the head and from far in the tail. They can be found on old spectra and the ion is probably very common as cometary plasma tails frequently show up on red-sensitive photographic plates, which should exclude the normal $\mathrm{CO}^{+}$emissions.

C. Arpigny of Liège (Institut d'Astrophysique) reported progress in the interpretation of molecular spectra, in particular in modelling collisional changes to the relative populations and line strengths of the purely resonant fluorescent description. Applying this potentially powerful method to the $\mathrm{CH}$ bands has given a reasonable total density in comet Bennett, with temperature of the colliding agent in the range $4-800^{\circ} \mathrm{K}$. Analyses of the extensive $\mathrm{H}$-coma were reported by $\mathrm{H}$. $\mathrm{U}$. Keller of LASP (University of Colorado), who has made this topic his own. All observations give outflow velocity in the range $7-10 \mathrm{~km}$, which may correspond to the as yet unknown surplus energy of $\mathrm{OH}$ photodissociation. But the distant Lyman- $\alpha$ profiles of comet Bennett, observed from OGO-5, require for their explanation an atomic $\mathrm{H}$ component of higher velocity, around $20 \mathrm{~km} \mathrm{~s}^{-1}$, as would be expected from the photodissociation of $\mathrm{H}_{2} \mathrm{O}$.

Including the new identifications. A. H. Delsemme (University of Toledo) advocated the simple two-stage picture of the cometary coma: $\mathrm{H}_{2} \mathrm{O}$ and certain unknown parent molecules expand radially outwards and dissociate into daughter radicals and atoms, which are subsequently destroyed by ionisation. He gave length scales for the hypothetical parents and observed daughters. But a paper by C. K. Kumar and R. J. Southall of Howard University reported widelv different scales in various comets for the supposed parents of $\mathrm{CN}$ and C.. And a chemist. R. Oppenheimer of Harvard. emphasised in a snirited contribution that numerous ion-molecule and dissociation-recombination processes can proceed rapidly inside 10.000 $\mathrm{km}$ in the coma. Various comnlex reaction chains could occur. resulting in $\mathrm{CH}_{3} \mathrm{CN}$. $\mathrm{C}_{3}$ and. in princinle, even $\mathrm{H} \cdot \mathrm{O}$ ! The fundamental constituents of comets are still very much in doubt.

H. U. Schmidt of Munich (MaxPlanck Institut für Physik und
Astrophysik) reviewed progress in the fluid description of plasma motion through the coma. He considered that magnetic stress from the distorted field of the solar wind would reduce the distance of the contact discontinuity dividing off the purely cometary plasma, but explanations of how structures were produced from this plasma and gave rise to the observed tail were still lacking. M. K. Wallis (University of Oxford) argued, however, that because of cooling and recombination processes, the 'contact discontinuity' would occur inside the $10,000 \mathrm{~km}$ collisional region and therefore have little significance.

Sequences of pictures of ion tails of various comets had been made into a film by K. Jockers (Sacramento Peak Observatory). This illustrated particularly that subsidiary systems of rays sometimes arise from condensations in the tail and that nearby condensations and wave features move at similar speeds, so would appear to be convected features in a general flow. Progress was being made in the theory of dust tails, reported Z. Sekanina (Smithsonian Astrophysical Observatory). The fact that the 'silicate signature' in the infrared emission is not present in the anti-tail proves the particles differ in size.

There was as ever speculation about processes of comet formation: A. Mendis (University of California, San Diego) suggested that they could be accreted on the time scale of $10^{5}$ years in present-day meteor streams: F. $\mathbf{L}$. Whipple (Smithsonian Astrophysical Observatory) wished to use the compression of a solar gale from the young sun, while B. Donn (NASA: Goddard Space Flight Center) reckoned comets might be formed in a dense cloud within the cluster from which the sun emerged.

As to the long discussed mission to a comet, a space probe to comet Encke in 1980 now has a reasonable chance, reported I. Rasool (NASA). With a 'slow fly-by at $8 \mathrm{~km} \mathrm{~s}^{-1}$, the nucleus could be photographed and the neutral coma and plasma coma and tail could be investigated.

It is aimed to publish both impromptu and scheduled talks, by courtesy of NASA. in what will surely be voluminous conference proceedings.

\section{Correction}

The source of the illustrations from the ceiling of the British Museum (Natural History) (Nature, 252, 193: 1974) was not acknowledged: they were reproduced from the Botany Leaflet No. 1 published by the British Museum (Natural History). 